\title{
Complex interactions between malaria and malnutrition: a systematic literature review
}

\author{
D Das ${ }^{1,2}$, R F Grais ${ }^{4}$, E A Okiro ${ }^{7}$, K Stepniewska ${ }^{1,2}$, R Mansoor ${ }^{1,2}$, S van der Kam5 , D J Terlouw ${ }^{6,10,11}$, J Tarning ${ }^{1,2,3}$, \\ KI Barnes ${ }^{8,9}$ and P J Guerin ${ }^{1,2^{*}}$
}

\begin{abstract}
Background: Despite substantial improvement in the control of malaria and decreased prevalence of malnutrition over the past two decades, both conditions remain heavy burdens that cause hundreds of thousands of deaths in children in resource-poor countries every year. Better understanding of the complex interactions between malaria and malnutrition is crucial for optimally targeting interventions where both conditions co-exist. This systematic review aimed to assess the evidence of the interplay between malaria and malnutrition.
\end{abstract}

Methods: Database searches were conducted in PubMed, Global Health and Cochrane Libraries and articles published in English, French or Spanish between Jan 1980 and Feb 2018 were accessed and screened. The methodological quality of the included studies was assessed using the Newcastle-Ottawa Scale and the risk of bias across studies was assessed using the GRADE approach. The preferred reporting items for systematic reviews and meta-analyses (PRISMA) guideline were followed.

Results: Of 2945 articles screened from databases, a total of 33 articles were identified looking at the association between malnutrition and risk of malaria and/or the impact of malnutrition in antimalarial treatment efficacy. Large methodological heterogeneity of studies precluded conducting meaningful aggregated data meta-analysis. Divergent results were reported on the effect of malnutrition on malaria risk. While no consistent association between risk of malaria and acute malnutrition was found, chronic malnutrition was relatively consistently associated with severity of malaria such as high-density parasitemia and anaemia. Furthermore, there is little information on the effect of malnutrition on therapeutic responses to artemisinin combination therapies (ACTs) and their pharmacokinetic properties in malnourished children in published literature.

Conclusions: The evidence on the effect of malnutrition on malaria risk remains inconclusive. Further analyses using individual patient data could provide an important opportunity to better understand the variability observed in publications by standardising both malaria and nutritional metrics. Our findings highlight the need to improve our understanding of the pharmacodynamics and pharmacokinetics of ACTs in malnourished children. Further clarification on malaria-malnutrition interactions would also serve as a basis for designing future trials and provide an opportunity to optimise antimalarial treatment for this large, vulnerable and neglected population.

Trial registration: PROSPERO CRD42017056934.

Keywords: Malaria, Anthropometry, Stunting, Wasting, Underweight

\footnotetext{
* Correspondence: philippe.guerin@wwarn.org

${ }^{1}$ WorldWide Antimalarial Resistance Network (WWARN), Oxford, UK

${ }^{2}$ Centre for Tropical Medicine and Global Health, Nuffield Department of

Clinical Medicine, University of Oxford, Oxford, UK

Full list of author information is available at the end of the article
}

(c) The Author(s). 2018 Open Access This article is distributed under the terms of the Creative Commons Attribution 4.0 International License (http://creativecommons.org/licenses/by/4.0/), which permits unrestricted use, distribution, and reproduction in any medium, provided you give appropriate credit to the original author(s) and the source, provide a link to the Creative Commons license, and indicate if changes were made. The Creative Commons Public Domain Dedication waiver (http://creativecommons.org/publicdomain/zero/1.0/) applies to the data made available in this article, unless otherwise stated. 


\section{Background}

Malaria and malnutrition in children (refers to all forms of undernutrition) are reported independently to be major causes of morbidity and mortality in low- and middle-income countries. About 3.2 billion people remained at risk of malaria with an estimated 216 million new cases (95\% confidence interval 196-263 million) and 445,000 deaths worldwide in 2016; the majority of deaths occur in children under 5 years in sub-Saharan Africa [1]. Approximately 3.1 million deaths in children under five are attributed to malnutrition each year, representing $45 \%$ of all childhood deaths [2]. The interaction between malaria and childhood malnutrition has been studied for many years and complex interactions between these high-burden conditions are now increasingly recognised. Understanding the consequences of malnutrition on malaria and vice versa is crucial and may help guide the choice of public health interventions and research priorities where significant co-morbidity exists.

Malnutrition is a complex phenomenon due to its multifactorial aetiology and diverse clinical presentation. Acute malnutrition manifests with wasting (low weight for height) and chronic malnutrition as stunting (low height for age). Being underweight (low weight for age) can result from either chronic or acute malnutrition or both. Assessment of malnourishment can be conducted using anthropometric indicators which compare child's weight and height to the standardised age- and sex-specific growth reference derived from the international reference population of children between 6 and 59 months of age (World Health Organization (WHO) Child Growth Standards 2006) [3]. The anthropometric indicators are expressed as a number of standard deviations (SDs) below or above the reference mean or median value, $\mathrm{Z}$-score. Cutoffs of -3 are used to indicate severe malnutrition and values between -2 and -3 are considered to be moderate malnutrition. Weight-for-height Z-score (WHZ) is the indicator used to classify a child with wasting. Mid-Upper Arm Circumference (MUAC) is another frequently used indicator for wasting. Severe acute malnutrition (SAM) is defined as MUAC $<115 \mathrm{~mm}$ and/or $\mathrm{WHZ}<-3$ and/or bilateral pitting oedema. Stunting is defined by the measure of height-for-age Z-score (HAZ) and a child is considered as being underweight based on low weight-for-age Z-score (WAZ). These definitions do not take micronutrient malnutrition into account, which can occur even if the person is getting enough energy and they are not thin or short.

The exact relationship between childhood malnutrition and malaria remains complex, controversial, and poorly understood. One of the key questions is, to what extent the burden of malaria is attributable to wasting and stunting? In regard to the impact of malaria on malnutrition, some evidence suggests malaria has adverse effects on nutritional status of young children [4-9]. On the other hand, whether and how malnutrition influences malaria morbidity and mortality is debated. Some studies have reported that malnutrition is associated with a higher risk of malaria [10-13], others have suggested a protective effect [14-17], or no differential risk $[18,19]$. Similarly, there is very limited evidence on the relationship between nutritional status and antimalarial drug efficacy. Clinical efficacy of the current first-line malaria treatment, the artemisinin combination therapies (ACTs), in malnourished children has been rarely explored [20-22]. Understanding the complex relationship of the immune response of individuals infected with malaria and suffering of malnutrition is crucial to guide specific antimalarial therapeutic approaches in this vulnerable sub-population. There are key knowledge gaps in defining the complex relationship between malnutrition and malaria, which need to be identified and addressed. We aimed to conduct a systematic review of the current understanding of interactions between acute or chronic malnutrition and the risk of developing malarial infection. A further objective was to explore published literature on the impact of malnutrition on the efficacy of antimalarial treatment.

\section{Methods}

We conducted a systematic literature review of manuscripts published between Jan 1, 1980, and Feb 19, 2018. PubMed, Global Health and Cochrane Libraries were searched using key terms (Additional file 1), and articles published in English, French or Spanish were accessed. Two reviewers identified relevant articles of interest, as per criteria listed below, by screening titles and abstracts of publications retrieved. The preferred reporting items for systematic reviews and meta-analyses (PRISMA) guideline [23] was followed. The PRISMA checklist is provided as Additional file 2 and the review is registered in international prospective register of systematic reviews (PROSPERO Registration No. CRD42017056934).

\section{Eligibility screening}

For the selected articles, full text was obtained and assessed for relevance to any of the following topics of interest: (1) association between malnutrition and risk of malaria and (2) malnutrition and antimalarial treatment efficacy. We excluded studies primarily focused on non-malarial conditions such as TB, HIV, neglected tropical diseases, pneumonia or diarrhoeal diseases; non-clinical studies (systematic reviews, opinion pieces, editorials, modelling studies, economic evaluations, guidelines, protocols, book chapters) and in vitro, animal, plant or molecular studies; studies on malaria in pregnancy and placental malaria; demographic and health surveys, mortality surveys, qualitative studies; case reports or series; vaccine studies; and 
studies primarily focused only on malaria or malnutrition or micronutrient deficiencies or anaemia. For this review, observational and interventional studies in non-pregnant populations with malnutrition assessed by anthropometric measurements as exposure and risk of malaria infection (whether asymptomatic parasitemia or uncomplicated malaria) as outcome were included.

\section{Data extraction}

From each of the included studies in this review, the following variables were extracted: authors, year of publication, country, study design, age (range or median/mean), sex (ratio), sample size, growth standards, malaria transmission intensity, definition of malaria and the reported risk estimates. The methodological quality of the included studies was assessed using the Newcastle-Ottawa Scale (NOS) [24] and the risk of bias across studies was assessed using the GRADE approach [25]. The risk of bias assessment within and across studies is presented as Additional file 3.

\section{Analysis}

Aggregated data meta-analysis was not possible due to the heterogeneity of studies in respect to study design, definition of malnutrition, definition of malaria, study population, e.g. age group target, analysis conducted and effect measures presented. Only summaries of study findings are presented in this review. Association between malnutrition and risk of malaria was deemed to be statistically significant if either the $P$ value was $<0.05$ and/or the $95 \%$ confidence intervals (CIs) did not include 1 . The risk of malaria was classified as "increased" or "decreased" according to the interpretation of the effect estimates provided (i.e. incidence risk ratio (IRR), odds ratio (OR), risk ratio (RR) or hazard ratio (HR)) if statistical significance was achieved as described above.

\section{Results}

The literature search identified 2945 articles. A total of 32 articles identified through the search and 1 article obtained through citation tracking were included, describing cross-sectional surveys $(n=16)$, longitudinal studies $(n=12)$, interventional studies $(n=3)$, case-control study $(n=1)$ and individual patient data meta-analysis $(n=1)$ (Fig. 1). Details of the 33 studies included in this review are given in Table 1, while the study characteristics are summarised in Additional file 4.

\section{Association between malnutrition and risk of malaria}

In total, 29 studies assessed the association between malnutrition and risk of malaria (Tables 2, 3 and 4).

\section{Risk of malaria infection in children with stunting (chronic malnutrition)}

Twenty-three studies explored the relationship between stunting and risk of malaria infection (Table 2). Overall results were conflicting, with 15 studies showing that

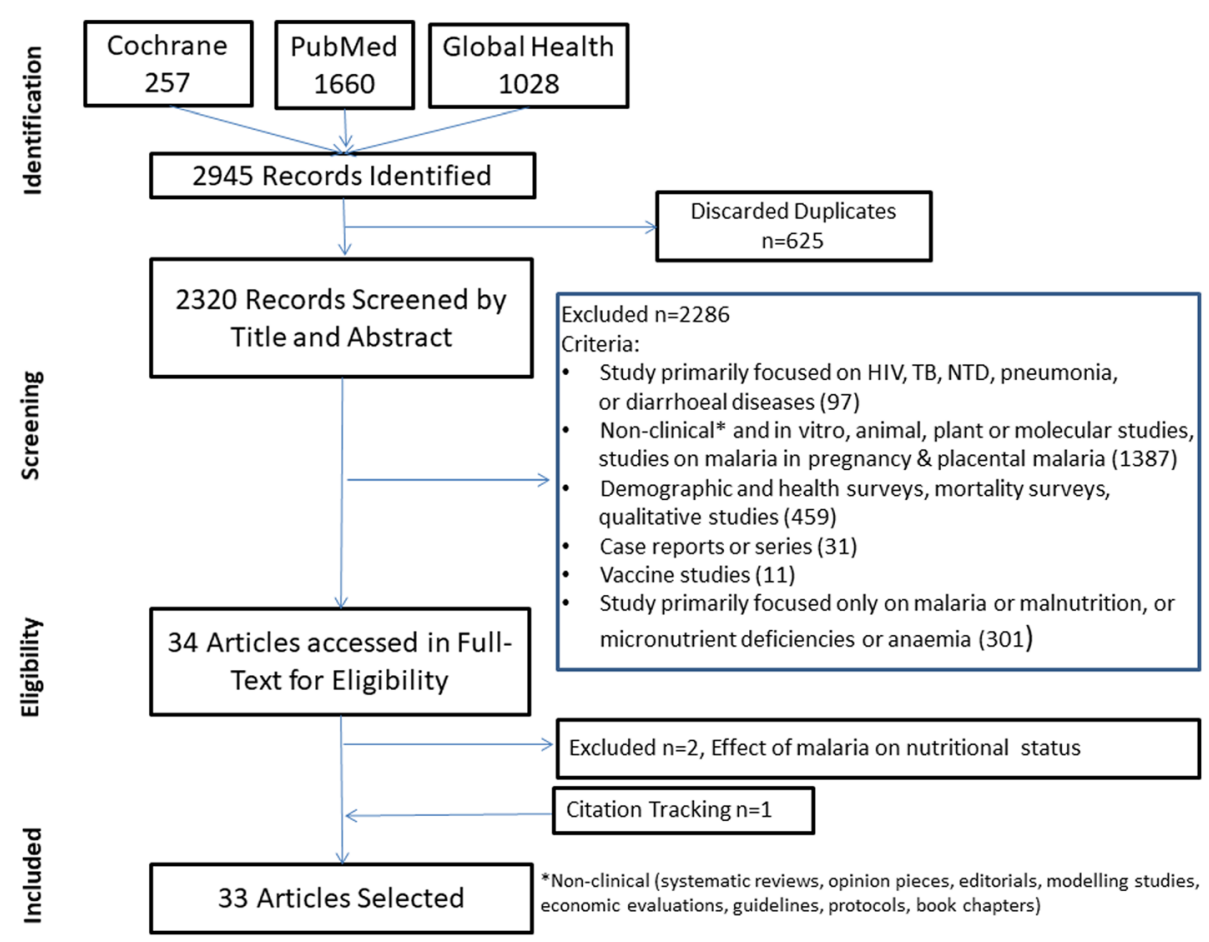

Fig. 1 Flow diagram of study selection 
Table 1 Details of the studies included $(N=33)$

\begin{tabular}{|c|c|c|c|c|}
\hline Author, year, reference & Country & Region & Study design & $\begin{array}{l}\text { Topics of } \\
\text { interest* }\end{array}$ \\
\hline Akiyama 2016 [46] & Lao PDR & $\begin{array}{l}\text { South East } \\
\text { Asia }\end{array}$ & Cross-sectional & $1 a, 1 c$ \\
\hline Alexandre 2015 [47] & Brazilian Amazon & $\begin{array}{l}\text { Latin } \\
\text { America }\end{array}$ & Longitudinal & $1 a$ \\
\hline Arinaitwe 2012 [48] & Uganda & Africa & Longitudinal & $1 \mathrm{a}, 1 \mathrm{c}$ \\
\hline Ayana 2015 [49] & Ethiopia & Africa & Retrospective cohort & $1 a, 1 b, 1 c$ \\
\hline Bilal Shikur 2016 [28] & Ethiopia & Africa & Case-control & $1 b$ \\
\hline Crookston 2010 [50] & Ghana & Africa & Cross-sectional & $1 a$ \\
\hline Custodio 2009 [30] & Equatorial Guinea & Africa & Survey & $1 a, 1 b, 1 c$ \\
\hline Deen 2002 [10] & Gambia & Africa & Longitudinal & $1 \mathrm{a}, 1 \mathrm{~b}, 1 \mathrm{c}$ \\
\hline Denoeud-Ndam 2016 [35] & Mali, Niger & Africa & $\begin{array}{l}\text { Non-randomised comparative } \\
\text { trial }\end{array}$ & 2 \\
\hline Deribew 2010 [51] & Ethiopia & Africa & Cross-sectional & $1 a, 1 b, 1 c$ \\
\hline Ehrhardt 2006 [11] & Ghana & Africa & Survey & $1 b, 1 c$ \\
\hline El Samani 1987 [52] & Sudan & Africa & Cross-sectional & 1c \\
\hline Fillol 2009 [27] & Senegal & Africa & Longitudinal & $1 \mathrm{a}, 1 \mathrm{~b}, 1 \mathrm{c}$ \\
\hline Friedman 2005 [26] & Kenya & Africa & Survey & $1 a, 1 b$ \\
\hline Genton 1998 [14] & Papua New Guinea & Oceania & Longitudinal & $1 \mathrm{a}, 1 \mathrm{~b}$ \\
\hline Jeremiah 2007 [53] & Nigeria & Africa & Cross-sectional & $1 c$ \\
\hline Kateera 2015 [54] & Rwanda & Africa & Cross-sectional & $1 a, 1 b, 1 c$ \\
\hline Maketa 2015 [55] & $\begin{array}{l}\text { Democratic Republic of Congo } \\
\text { (DRC) }\end{array}$ & Africa & Cross-sectional & $1 a$ \\
\hline Mamiro 2005 [56] & Tanzania & Africa & Cross-sectional & $1 a$ \\
\hline Mitangala 2012 [34] & $\begin{array}{l}\text { Democratic Republic of Congo } \\
\text { (DRC) }\end{array}$ & Africa & Therapeutic efficacy study & 2 \\
\hline Mitangala 2013 [21] & $\begin{array}{l}\text { Democratic Republic of Congo } \\
\text { (DRC) }\end{array}$ & Africa & Survey & $1 a, 1 b, 1 c$ \\
\hline Muller 2003 [18] & Burkina Faso & Africa & Longitudinal & $1 \mathrm{a}, 1 \mathrm{~b}, 1 \mathrm{c}$ \\
\hline Nyakeriga 2004 [6] & Kenya & Africa & Longitudinal & $1 \mathrm{a}, 1 \mathrm{c}$ \\
\hline Obua 2008 [33] & Uganda & Africa & Therapeutic efficacy study & 2 \\
\hline Snow 1991 [17] & Gambia & Africa & Longitudinal & $1 a, 1 b, 1 c$ \\
\hline Sumbele 2015 [57] & Cameroon & Africa & Cross-sectional & $1 \mathrm{a}, 1 \mathrm{~b}, 1 \mathrm{c}$ \\
\hline Takakura 2001 [29] & Lao PDR & $\begin{array}{l}\text { South East } \\
\text { Asia }\end{array}$ & Survey & $1 b$ \\
\hline Tonglet 1999 [32] & $\begin{array}{l}\text { Democratic Republic of Congo } \\
\text { (DRC) }\end{array}$ & Africa & Longitudinal & $1 \mathrm{a}, 1 \mathrm{c}$ \\
\hline Uscategui Penuela 2009 [58] & Colombia & $\begin{array}{l}\text { Latin } \\
\text { America }\end{array}$ & Cross-sectional & $1 \mathrm{a}, 1 \mathrm{~b}$ \\
\hline Verhoef 2002 [13] & Kenya & Africa & Survey & $1 \mathrm{a}, 1 \mathrm{~b}$ \\
\hline Verret 2011 [22] & Uganda & Africa & Longitudinal & $1 \mathrm{a}, 1 \mathrm{c}, 2$ \\
\hline William 1997 [31] & Vanuatu & Oceania & Longitudinal & $1 b, 1 c$ \\
\hline $\begin{array}{l}\text { WWARN Lumefantrine PK/PD Study Group } \\
2015 \text { [36] }\end{array}$ & Multiple & Multiple & $\begin{array}{l}\text { Individual patient data meta- } \\
\text { analysis }\end{array}$ & 2 \\
\hline
\end{tabular}

*Topics of Interest: Risk of malaria infection in children with (1a) stunting, (1b) wasting, (1c) underweight; (2) malnutrition and anti-malarial drug efficacy 
Table 2 Relationship between chronic malnutrition (stunting) and risk of malarial infection $(N=23)$

\begin{tabular}{|c|c|c|c|c|}
\hline $\begin{array}{l}\text { Author, Year, } \\
\text { Reference }\end{array}$ & HAZ cutoff & Malaria outcome & $\begin{array}{l}\text { Risk estimate comparing children below and } \\
\text { above HAZ cutoff }\end{array}$ & Risk \\
\hline Akiyama 2016 [46] & $\leq-2$ & Asymptomatic malaria confirmed by PCR & $\mathrm{OR}=3.34(95 \% \mathrm{Cl}=1.25-8.93)$ & Increased \\
\hline Alexandre 2015 [47] & $<-2$ & Fever and thick blood smear & $\mathrm{HR}=0.31(95 \% \mathrm{Cl}=0.10-0.99), P=0.049$ & Decreased \\
\hline \multirow[t]{2}{*}{ Arinaitwe 2012 [48] } & -1 and -2 & Pf malaria, fever and a positive blood smear & $\operatorname{IRR}=1.24(95 \% \mathrm{Cl} 1.06-1.46), P=0.008$ & Increased \\
\hline & $<-2$ & Pf malaria, fever and a positive blood smear & $\mathrm{IRR}=1.24(95 \% \mathrm{Cl} 1.03-1.48), P=0.02$ & Increased \\
\hline Ayana 2015 [49] & $<-2$ & Malaria by RDT & $\mathrm{HR}=2.50(95 \% \mathrm{Cl}=1.4-5.1)$ & Increased \\
\hline \multirow[t]{3}{*}{ Crookston 2010 [50] } & $\begin{array}{l}\geq-2 \text { and } \\
<-1\end{array}$ & Asymptomatic malaria confirmed by PCR & $\mathrm{OR}=2.23(95 \% \mathrm{Cl}=0.99-5.02)$ & $\begin{array}{l}\text { No } \\
\text { impact }\end{array}$ \\
\hline & $\begin{array}{l}\geq-3 \text { and } \\
<-2\end{array}$ & Asymptomatic malaria confirmed by PCR & $\mathrm{OR}=0.56(95 \% \mathrm{Cl}=0.16-1.69)$ & $\begin{array}{l}\text { No } \\
\text { impact }\end{array}$ \\
\hline & $<-3$ & Asymptomatic malaria confirmed by PCR & $\mathrm{OR}=1.02(95 \% \mathrm{Cl}=0.20-3.76)$ & $\begin{array}{l}\text { No } \\
\text { impact }\end{array}$ \\
\hline Custodio 2009 [30] & $<-2$ & Pf malaria parasitemia prevalence & $\mathrm{OR}=3.07(95 \% \mathrm{Cl}=1.40-6.73)$ & Increased \\
\hline Deen 2002 [10] & $<-2$ & $\begin{array}{l}\text { Malaria episode (fever } \geq 37.5^{\circ} \mathrm{C} \text { or parasitemia } \\
>5000 / \mu \mathrm{L} \text { ) }\end{array}$ & $\mathrm{RR}=1.35(95 \% \mathrm{Cl}=1.08-1.69), P=0.01$ & Increased \\
\hline Deribew 2010 [51] & $<-2$ & Pf malaria (any parasitemia) & $\mathrm{AOR}=0.9(95 \% \mathrm{Cl}=0.7-1.2), P=0.85$ & $\begin{array}{l}\text { No } \\
\text { impact }\end{array}$ \\
\hline \multirow[t]{2}{*}{ Fillol 2009 [27] } & $<-2$ & $\begin{array}{l}\text { Clinical malaria (fever } \geq 37.5^{\circ} \mathrm{C} \text { plus parasitemia } \\
\geq 3000 / \mu \mathrm{L} \text { ) }\end{array}$ & "Non-significant association" reported & $\begin{array}{l}\text { No } \\
\text { impact }\end{array}$ \\
\hline & $<-2$ & $\begin{array}{l}\text { High density parasitemia (geometric mean } \geq \\
300 / \mu \mathrm{L} \text { ) }\end{array}$ & $\mathrm{AOR}=2.42(95 \% \mathrm{Cl}=1.12-5.24), P=0.03$ & Increased \\
\hline \multirow[t]{4}{*}{ Friedman 2005 [26] } & $<-2$ & Concurrent malaria (any parasitemia) & $\mathrm{AOR}=1.98, P<0.0001$ & Increased \\
\hline & $<-2$ & $\begin{array}{l}\text { High density parasitemia (any species, > 1500- } \\
7000 / \mu \mathrm{L} \text { ) }\end{array}$ & $\mathrm{AOR}=1.84, P<0.0001$ & Increased \\
\hline & $<-2$ & $\begin{array}{l}\text { Clinical malaria (fever plus high density } \\
\text { parasitemia) }\end{array}$ & $\mathrm{AOR}=1.77, P=0.06$ & Increased \\
\hline & $<-2$ & Severe anaemia (Haemoglobin < 7 g/dL) & $\mathrm{AOR}=2.65, P<0.0001^{*}$ & - \\
\hline \multirow[t]{3}{*}{ Genton 1998 [14] } & $<-2$ & Pf malaria (fever plus any parasitemia) & Adj. Rate ratio $=1.13(95 \% \mathrm{Cl}=0.98-1.29), P=0.09$ & $\begin{array}{l}\text { No } \\
\text { impact }\end{array}$ \\
\hline & $<-2$ & Pf malaria (fever plus parasitemia $\geq 5 \times 10^{9} / \mathrm{L}$ ) & Adj. Rate ratio $=1.19(95 \% \mathrm{Cl}=1.01-1.40), P=0.03$ & Increased \\
\hline & $<-2$ & Pf malaria (fever plus parasitemia $\geq 10 \times 10^{9} / \mathrm{L}$ ) & Adj. Rate ratio $=1.18(95 \% \mathrm{Cl}=0.98-1.41), P=0.08$ & $\begin{array}{l}\text { No } \\
\text { impact }\end{array}$ \\
\hline Kateera 2015 [54] & $<-2$ & Pf malaria (any parasitemia) & "Non-significant association" reported & $\begin{array}{l}\text { No } \\
\text { impact }\end{array}$ \\
\hline Maketa 2015 [55] & $\leq-2$ & Asymptomatic malaria by blood smear & $\mathrm{AOR}=1.8, P=0.01$ & Increased \\
\hline Mamiro 2005 [56] & $<-2$ & Malaria by blood smear & $\mathrm{AOR}=1.9(95 \% \mathrm{Cl}=1.1-3.2), P=0.02$ & Increased \\
\hline \multirow[t]{2}{*}{ Mitangala 2013 [21] } & $<-2$ & Pf malaria parasitemia ( $\geq 5000 / \mu \mathrm{L})$ & $\mathrm{AOR}=0.72(95 \% \mathrm{Cl}=0.37-1.40)$ & $\begin{array}{l}\text { No } \\
\text { impact }\end{array}$ \\
\hline & $<-3$ & Pf malaria parasitemia ( $\geq 5000 / \mu \mathrm{L})$ & $\mathrm{AOR}=0.48(95 \% \mathrm{Cl}=0.25-0.91)$ & Decreased \\
\hline \multirow[t]{3}{*}{ Muller 2003 [18] } & $\leq-2$ & Pf malaria (fever plus parasitemia $\geq 1 / \mu \mathrm{L}$ ) & $\mathrm{RR}=1.0(95 \% \mathrm{Cl}=0.9-1.1), P=0.87$ & $\begin{array}{l}\text { No } \\
\text { impact }\end{array}$ \\
\hline & $\leq-2$ & Pf malaria (fever plus parasitemia $\geq 5000 / \mu \mathrm{L}$ ) & $\mathrm{RR}=1.0(95 \% \mathrm{Cl}=0.9-1.2), P=0.59$ & $\begin{array}{l}\text { No } \\
\text { impact }\end{array}$ \\
\hline & $\leq-2$ & Pf malaria (fever plus parasitemia $\geq 100,000 / \mu \mathrm{L}$ ) & $\mathrm{RR}=0.8(95 \% \mathrm{Cl}=0.5-1.4), P=0.44$ & $\begin{array}{l}\text { No } \\
\text { impact }\end{array}$ \\
\hline \multirow[t]{2}{*}{ Nyakeriga 2004 [6] } & $<-2$ & Pf malaria (fever plus any parasitemia) & Adj. IRR $=1.89(95 \% \mathrm{Cl}=1.01-3.53), P=0.05$ & Increased \\
\hline & $<-2$ & Pf malaria (fever plus any para $<1$ year, $>$ & Adj. IRR $=1.93(95 \% \mathrm{Cl}=0.9-4.16), P=0.09$ & Increased \\
\hline
\end{tabular}


Table 2 Relationship between chronic malnutrition (stunting) and risk of malarial infection $(N=23)$ (Continued)

\begin{tabular}{|c|c|c|c|c|}
\hline $\begin{array}{l}\text { Author, Year, } \\
\text { Reference }\end{array}$ & HAZ cutoff & Malaria outcome & $\begin{array}{l}\text { Risk estimate comparing children below and } \\
\text { above HAZ cutoff }\end{array}$ & Risk \\
\hline \multirow[t]{2}{*}{ Snow 1991 [17] } & $<-2$ & Clinical malaria (fever plus any parasitemia) & "Non-significant association" reported & $\begin{array}{l}\text { No } \\
\text { impact }\end{array}$ \\
\hline & $<-2$ & Asymptomatic malaria parasitemia & "Non-significant association" reported & $\begin{array}{l}\text { No } \\
\text { impact }\end{array}$ \\
\hline \multirow[t]{2}{*}{ Sumbele 2015 [57] } & $<-2$ & Clinical malaria parasitemia & Stunted vs. Non-stunted: $16.9 \%$ vs. $7.5 \%, P=0.01$ & Increased \\
\hline & $<-2$ & Asymptomatic malaria parasitemia & Stunted vs. Non-stunted: $26.0 \%$ vs. $26.2 \%, P=0.91$ & $\begin{array}{l}\text { No } \\
\text { impact }\end{array}$ \\
\hline \multirow[t]{2}{*}{ Tonglet 1999 [32] } & $<-2$ & $\begin{array}{l}\text { Clinical malaria without lab confirmation in < } \\
9 \mathrm{~m}\end{array}$ & $\mathrm{AOR}=1.16(95 \% \mathrm{Cl}=0.54-1.77)$ & Increased \\
\hline & $<-2$ & $\begin{array}{l}\text { Clinical malaria without lab confirmation in } \geq \\
9 \mathrm{~m}\end{array}$ & $\mathrm{AOR}=0.71(95 \% \mathrm{Cl}=0.28-1.14)$ & $\begin{array}{l}\text { No } \\
\text { impact }\end{array}$ \\
\hline $\begin{array}{l}\text { Uscategui Penuela } \\
2009 \text { [58] }\end{array}$ & $<-2$ & Malaria infection & $\mathrm{OR}=1.94(95 \% \mathrm{Cl}=1.07-3.50), P=0.023$ & Increased \\
\hline Verhoef 2002 [13] & $<-2$ & Laboratory confirmed malaria & $\mathrm{OR}=0.87(95 \% \mathrm{Cl}=0.69-1.09), P=0.23$ & $\begin{array}{l}\text { No } \\
\text { impact }\end{array}$ \\
\hline \multirow[t]{3}{*}{ Verret 2011 [22] } & $\begin{array}{l}\geq-1 \text { and } \\
<0\end{array}$ & Pf malaria risk of recurrent parasitemia & $\mathrm{HR}=2.35(95 \% \mathrm{Cl}=0.85-6.48), P=0.099$ & $\begin{array}{l}\text { No } \\
\text { impact }\end{array}$ \\
\hline & $\begin{array}{l}\geq-2 \text { and }< \\
-1\end{array}$ & Pf malaria risk of recurrent parasitemia & $\mathrm{HR}=2.89(95 \% \mathrm{Cl}=1.06-7.89), P=0.039$ & Increased \\
\hline & $<-2$ & Pf malaria risk of recurrent parasitemia & $\mathrm{HR}=3.18(95 \% \mathrm{Cl}=1.18-8.56), P=0.022$ & Increased \\
\hline
\end{tabular}

stunting was associated with an increased malaria risk, 11 studies showing no association and 2 studies showing a protective effect of stunting (Table 2).

A prospective cohort study of 487 children under 5 years of age in rural Gambia by Deen et al. reported that being stunted increased the risk of malaria infection significantly $(\mathrm{RR}=1.35 \quad(95 \% \quad \mathrm{CI}=1.08-1.69)) \quad[10] . \quad$ The authors hypothesised that the observed association between malnutrition and malaria infection might be influenced by confounding factors such as HIV co-infection or socio-economic factors. Similarly, in a cross-sectional survey in children $<3$ years in Kenya, Friedman and colleagues found an increased malaria risk in stunted children, showing a trend towards an increased risk of clinical malaria $(\mathrm{OR}=1.77, P=0.06)$, and significantly increased risk of any malaria parasitemia $(\mathrm{OR}=1.98, P<0.0001)$, high-density parasitemia (any species, $>1500-7000 / \mu \mathrm{L}$; $\mathrm{OR}=1.84, P<0.0001)$ and severe anaemia $(\mathrm{OR}=2.65, P<$ 0.0001 ) [26]. In contrast, a longitudinal study from the Gambia by Snow et al. in children aged $1-4$ years reported only a minor (non-significant) impact of stunting on clinical and asymptomatic malaria episodes [17]. Two longitudinal malaria surveillance reports, one in Senegal with 874 children aged 12 months -5 years and the other in Burkina Faso with 685 children aged 6-30 months did not show any association between a low HAZ and subsequent malaria attacks [18, 27]. Similarly, Verhoef et al. in Kenya did not observe an association between being stunted and the risk of malaria infection; however, they showed that stunting might determine the severity of malaria-associated anaemia in African children [13]. Verret et al. found that in chronically malnourished children in a high-transmission setting in Uganda, children with mild (HAZ $[\geq-2$ and $<-1]$ ) to moderate (HAZ <-2) stunting not given trimethoprimsulfamethoxazole prophylaxis were at higher risk for recurrent parasitemia [22]. Contrary to this, in a cohort survey of 790 children under 5 years in the Kivu province, Democratic Republic of Congo (DRC), Mitangala and colleagues found that being severely stunted was protective of subsequent malaria parasitemia [21]. This finding was supported by Genton et al. in a prospective cohort of 136 children aged 10-120 months in Papua New Guinea showing that lower HAZ had a protective effect against falciparum malaria [14].

\section{Risk of malaria infection in children with wasting}

Eighteen studies explored the relationship between wasting and risk of malaria infection (Table 3). Overall, results were again conflicting, with three studies showing that wasting was associated with an increased malaria risk, two studies showing a protective effect and most studies showing no association (Table 3 ).

Takakura et al. [29], Ehrhardt et al. [11] and Shikur et al. [28] found an increased risk of $P$. falciparum malaria in children with wasting. In a case-control study involving 428 under-five children in Ethiopia, Shikur and colleagues found that severely wasted children were three 
Table 3 Relationship between acute malnutrition (wasting) and risk of malarial infection ( $N=18)$

\begin{tabular}{|c|c|c|c|c|}
\hline $\begin{array}{l}\text { Author, Year, } \\
\text { Reference }\end{array}$ & $\begin{array}{l}\text { WHZ } \\
\text { cutoff }\end{array}$ & Malaria outcome & $\begin{array}{l}\text { Risk estimate comparing children below and above } \\
\text { WHZ cutoff }\end{array}$ & Risk \\
\hline Ayana 2015 [49] & $<-2$ & Malaria by RDT & "Non-significant association" reported & $\begin{array}{l}\text { No } \\
\text { impact }\end{array}$ \\
\hline \multirow[t]{2}{*}{ Bilal Shikur 2016 [28] } & $<-2$ & Malaria by RDT or blood film & $\mathrm{AOR}=0.66(95 \% \mathrm{Cl}=0.21-2.03)$ & $\begin{array}{l}\text { No } \\
\text { impact }\end{array}$ \\
\hline & $<-3$ & Malaria by RDT or blood film & $\mathrm{AOR}=2.90(95 \% \mathrm{Cl}=1.14-7.61), P=0.025$ & Increased \\
\hline Custodio 2009 [30] & $<-2$ & Pf malaria parasitemia prevalence & "Non-significant association" reported & $\begin{array}{l}\text { No } \\
\text { impact }\end{array}$ \\
\hline Deen 2002 [10] & $<-2$ & $\begin{array}{l}\text { Malaria episode (fever } \geq 37.5^{\circ} \mathrm{C} \text { or parasitemia } \\
>5000 / \mu \mathrm{L} \text { ) }\end{array}$ & $\mathrm{RR}=0.87(95 \% \mathrm{Cl}=0.69-1.10)$ & $\begin{array}{l}\text { No } \\
\text { impact }\end{array}$ \\
\hline Deribew 2010 [51] & $<-2$ & Pf malaria (any parasitemia) & $\mathrm{AOR}=0.6(95 \% \mathrm{Cl}=0.2-1.3), P=0.18$ & $\begin{array}{l}\text { No } \\
\text { impact }\end{array}$ \\
\hline \multirow[t]{2}{*}{ Ehrhardt 2006 [11] } & $<-2$ & Fever & $\mathrm{OR}=1.74(95 \% \mathrm{Cl}=1.16-2.60), P=0.004$ & . \\
\hline & $<-2$ & $\begin{array}{l}\text { Clinical malaria (fever } \geq 37.5^{\circ} \mathrm{C} \text { plus any } \\
\text { parasitemia) }\end{array}$ & $\mathrm{OR}=1.86(95 \% \mathrm{Cl}=1.14-3.02), P=0.007$ & Increased \\
\hline \multirow[t]{2}{*}{ Fillol 2009 [27] } & $<-2$ & $\begin{array}{l}\text { Clinical malaria (fever } \geq 37.5^{\circ} \mathrm{C} \text { plus parasitemia } \\
\geq 3000 / \mu \mathrm{L} \text { ) }\end{array}$ & $\mathrm{OR}=0.33(95 \% \mathrm{Cl}=0.13-0.81), P=0.02$ & Decreased \\
\hline & $<-2$ & $\begin{array}{l}\text { High-density parasitemia (geometric mean } \geq \\
300 / \mu \mathrm{L} \text { ) }\end{array}$ & $\mathrm{AOR}=0.48(95 \% \mathrm{Cl}=0.04-5.34), P=0.55$ & $\begin{array}{l}\text { No } \\
\text { impact }\end{array}$ \\
\hline \multirow[t]{4}{*}{ Friedman 2005 [26] } & $<-2$ & Concurrent malaria (any parasitemia) & $\mathrm{AOR}=0.75, P=0.18$ & $\begin{array}{l}\text { No } \\
\text { impact }\end{array}$ \\
\hline & $<-2$ & $\begin{array}{l}\text { High-density parasitemia (any species, > 1500- } \\
\text { 7000/ } \mathrm{L} \text { ) }\end{array}$ & $\mathrm{AOR}=0.96, P=0.88$ & $\begin{array}{l}\text { No } \\
\text { impact }\end{array}$ \\
\hline & $<-2$ & $\begin{array}{l}\text { Clinical malaria (fever plus high-density } \\
\text { parasitemia) }\end{array}$ & $\mathrm{AOR}=1.11, P=0.86$ & $\begin{array}{l}\text { No } \\
\text { impact }\end{array}$ \\
\hline & $<-2$ & Severe anaemia (Haemoglobin $<7 \mathrm{~g} / \mathrm{dL}$ ) & $\mathrm{AOR}=2.00, P=0.04^{*}$ & \\
\hline \multirow[t]{3}{*}{ Genton 1998 [14] } & $<-2$ & Pf malaria (fever plus any parasitemia) & Adj. rate ratio $=0.92(95 \% \mathrm{Cl}=0.77-1.11), P=0.4$ & $\begin{array}{l}\text { No } \\
\text { impact }\end{array}$ \\
\hline & $<-2$ & Pf malaria (fever plus parasitemia $\geq 5 \times 10^{9} / \mathrm{L}$ ) & Adj. rate ratio $=0.96(95 \% \mathrm{Cl}=0.77-1.19), P=0.69$ & $\begin{array}{l}\text { No } \\
\text { impact }\end{array}$ \\
\hline & $<-2$ & Pf malaria (fever plus parasitemia $\geq 10 \times 10^{9} / \mathrm{L}$ ) & Adj. rate ratio $=0.97(95 \% \mathrm{Cl}=0.75-1.24), P=0.78$ & $\begin{array}{l}\text { No } \\
\text { impact }\end{array}$ \\
\hline Kateera 2015 [54] & $<-2$ & Pf malaria (any parasitemia) & "Non-significant association" reported & $\begin{array}{l}\text { No } \\
\text { impact }\end{array}$ \\
\hline Mitangala 2013 [21] & $<-2$ & Pf malaria parasitemia ( $\geq 5000 / \mu \mathrm{L})$ & $\mathrm{AOR}=0.34(95 \% \mathrm{Cl}=0.08-1.45), P=0.15$ & $\begin{array}{l}\text { No } \\
\text { impact }\end{array}$ \\
\hline \multirow[t]{3}{*}{ Muller 2003 [18] } & $<-2$ & Pf malaria (fever plus parasitemia $\geq 1 / \mu \mathrm{L}$ ) & $\mathrm{RR}=1.0(95 \% \mathrm{Cl}=0.9-1.2), P=0.99$ & $\begin{array}{l}\text { No } \\
\text { impact }\end{array}$ \\
\hline & $<-2$ & Pf malaria (fever plus parasitemia $\geq 5000 / \mu \mathrm{L}$ ) & $\mathrm{RR}=1.0(95 \% \mathrm{Cl}=0.9-1.2), P=0.58$ & $\begin{array}{l}\text { No } \\
\text { impact }\end{array}$ \\
\hline & $<-2$ & Pf malaria (fever plus parasitemia $\geq 100,000 / \mu \mathrm{L}$ ) & $\mathrm{RR}=1.0(95 \% \mathrm{Cl}=0.5-1.8), P=0.94$ & $\begin{array}{l}\text { No } \\
\text { impact }\end{array}$ \\
\hline \multirow[t]{2}{*}{ Snow 1991 [17] } & $<-2$ & Clinical malaria (fever plus any parasitemia) & "Non-significant association" reported & $\begin{array}{l}\text { No } \\
\text { impact }\end{array}$ \\
\hline & $<-2$ & Asymptomatic malaria parasitemia & "Non-significant association" reported & $\begin{array}{l}\text { No } \\
\text { impact }\end{array}$ \\
\hline \multirow[t]{2}{*}{ Sumbele 2015 [57] } & $<-2$ & Clinical malaria parasitemia & Wasted vs. Non-wasted: $6.5 \%$ vs. $9.7 \%, P=0.78$ & $\begin{array}{l}\text { No } \\
\text { impact }\end{array}$ \\
\hline & $<-2$ & Asymptomatic malaria parasitemia & Wasted vs. Non-wasted: $22.6 \%$ vs. $26.7 \%, P=0.77$ & $\begin{array}{l}\text { No } \\
\text { impact }\end{array}$ \\
\hline \multirow[t]{2}{*}{ Takakura 2001 [29] } & $<-2$ & Pf malaria & Wasted vs. Non-wasted: $17 \%$ vs. $4 \%, P<0.05$ & Increased \\
\hline & $<-2$ & P. vivax malaria & "Non-significant association" reported & $\begin{array}{l}\text { No } \\
\text { impact }\end{array}$ \\
\hline
\end{tabular}


Table 3 Relationship between acute malnutrition (wasting) and risk of malarial infection $(N=18)$ (Continued)

\begin{tabular}{|c|c|c|c|c|}
\hline $\begin{array}{l}\text { Author, Year, } \\
\text { Reference }\end{array}$ & $\begin{array}{l}\text { WHZ } \\
\text { cutoff }\end{array}$ & Malaria outcome & $\begin{array}{l}\text { Risk estimate comparing children below and above } \\
\text { WHZ cutoff }\end{array}$ & Risk \\
\hline $\begin{array}{l}\text { Uscategui Penuela } \\
2009 \text { [58] }\end{array}$ & $<-2$ & Malaria infection & $\mathrm{OR}=2.64(95 \% \mathrm{Cl}=0.30-23.02), P=0.38$ & $\begin{array}{l}\text { No } \\
\text { impact }\end{array}$ \\
\hline Verhoef 2002 [13] & $<-2$ & Laboratory confirmed malaria & $\mathrm{OR}=0.78(95 \% \mathrm{Cl}=0.58-1.05), P=0.1$ & $\begin{array}{l}\text { No } \\
\text { impact }\end{array}$ \\
\hline \multirow[t]{2}{*}{ William 1997 [31] } & $<-2$ & Clinical malaria (fever plus para $\geq 1000 / \mu \mathrm{L}$ ) & "Non-significant association" reported & $\begin{array}{l}\text { No } \\
\text { impact }\end{array}$ \\
\hline & $<-2$ & P. vivax malaria & "Non-significant association" reported & $\begin{array}{l}\text { No } \\
\text { impact }\end{array}$ \\
\hline
\end{tabular}

Pf Plasmodium falciparum, WHZ weight-for-height Z-scores, $\mathrm{Cl}$ confidence interval, OR odds ratio, $H R$ hazard ratio, $R R$ risk ratio, IRR incidence rate ratio, $A O R$ adjusted odds ratio

*Limited to anaemia

times more likely to have malaria episode than non-wasted children (adjusted OR $=2.90$ (95\% CI $=1.14-7.61)$ [28]. In 2006, Ehrhardt et al. reported a survey involving 2905 children in Ghana aged 6-108 months in which wasting was significantly associated with a higher risk of clinical malaria $(\mathrm{OR}=1.86,95 \% \mathrm{CI}=1.14-3.02)$ [11]. Takakura et al. in a cross-sectional study of 309 children and adolescents (aged 2 to 18 years) in the Lao PDR showed that $P$. falciparum infection was associated with wasting [29]. However, Fillol and colleagues reported a significant protective association between being wasted (WHZ $<-2)$ at the onset of the rainy season and the risk of a clinical malaria episode $(\mathrm{OR}=0.33,95 \% \mathrm{CI}=0.13-0.81)$ in 874 preschool children (between 12 months and 5 years of age) in Senegal [27]. Similarly, in a cross-sectional survey of 1862 very young children (from 0 to 36 months age) in western Kenya, Friedman et al. showed that wasting decreased the risk of concurrent malaria $(\mathrm{OR}=0.75, P=0.18)$ and high-density parasitemia $(\mathrm{OR}=0.96, P=0.88)$, although increased the risk of severe malarial anaemia $(\mathrm{OR}=2.0, P=$ 0.04) [26]. In contrast, two other longitudinal studies conducted in the Gambia [17] and in Burkina Faso [18] and a few cross-sectional surveys from Equatorial Guinea [30], Eastern Kenya [13] and Ghana [11] reported no association between being wasted and the risk of malaria infections.

\section{Risk of malaria infection in underweight children}

Nineteen studies explored the relationship between being underweight-for-age and risk of malaria infection (Table 4). Overall, results were again conflicting, with five studies showing that underweight children carried a higher malaria risk, and the remaining studies showing no association (Table 4).

In 2006, Ehrhardt et al. using cross-sectional surveys in Ghana found that being underweight was significantly associated with a higher risk of having fever of any cause $(\mathrm{OR}=1.59,95 \% \mathrm{CI}=1.13-2.23)$, clinical malaria $(\mathrm{OR}=$ 1.67, $95 \% \mathrm{CI}=1.10-2.50)$ and anaemia $(\mathrm{OR}=1.68,95 \%$ $\mathrm{CI}=1.38-2.04)[11]$. This was confirmed by Sumbele et al. [57] who found that $21.6 \%$ of underweight children but only $8.2 \%$ of adequately nourished children developed clinical malaria $(P=0.007)$ in Cameroon. In a series of cross-sectional surveys conducted in the South Pacific island of Vanuatu in 1997, Williams et al. found a strong association between the incidence of $P$. vivax malaria and subsequently becoming underweight (IRR $=2.6,95 \%$ $\mathrm{CI}=1.5-4.4$ ) but no significant effect of $P$. falciparum malaria (IRR $=1.1,95 \% \mathrm{CI}=0.57-2.1)$ [31]. On the other hand, Tonglet et al. reported a non-significant protective association between being underweight and the risk of clinical malaria in children between 9 months and 2 years of age in the DRC $(\mathrm{OR}=0.68,95 \% \mathrm{CI}=0.24-1.11)$ [32].

\section{Malnutrition and anti-malarial drug efficacy}

Limited data exist on the effect of malnutrition on response to antimalarial drugs, in particular ACTs. Only five studies were identified in our literature search, and results were again contradictory.

In 2008, Obua et al. explored the impact of nutritional status on the dose, drug concentrations and treatment outcome with co-packaged chloroquine plus sulfadoxine-pyrimethamine in 83 children (6 months5 years) with uncomplicated falciparum malaria [33]. The authors found that stunting (height-for-age Z-score $<-2$ ) was associated with higher bodyweight-adjusted $(\mathrm{mg} / \mathrm{kg})$ doses of chloroquine and sulfadoxine-pyrimethamine, higher sulfadoxine concentrations on day 1 and chloroquine concentrations on day 3 , and better cure rates $(P=0.046)$.

In a longitudinal study of 292 infants (aged 412 months) in Uganda, a high malaria transmission intensity setting, ACTs (artemether-lumefantrine and dihydroartemisinin-piperaquine) were generally efficacious with a good early parasitological response ( $99 \%$ of study participants cleared parasites by day 3 ) for treatment of $P$. falciparum malaria, including in $43 \%$ chronically malnourished children [22]. However, in this study, stunted children (height-for-age Z-score $<-2$ ) in the dihydroartemisinin-piperaquine arm who were not taking trimethoprim-sulfamethoxazole prophylaxis (given to all 
Table 4 Relationship between being underweight and risk of malarial infection $(N=19)$

\begin{tabular}{|c|c|c|c|c|}
\hline $\begin{array}{l}\text { Author, year, } \\
\text { reference }\end{array}$ & WAZ cutoff & Malaria outcome & $\begin{array}{l}\text { Risk estimate comparing children below and } \\
\text { above WAZ cutoff }\end{array}$ & Risk \\
\hline $\begin{array}{l}\text { Akiyama } 2016 \\
{[46]}\end{array}$ & $\leq-2$ & Asymptomatic malaria confirmed by PCR & $\mathrm{OR}=1.33(95 \% \mathrm{Cl}=0.53-3.30)$ & $\begin{array}{l}\text { No } \\
\text { impact }\end{array}$ \\
\hline \multirow[t]{2}{*}{$\begin{array}{l}\text { Arinaitwe } 2012 \\
\text { [48] }\end{array}$} & -1 and -2 & Pf malaria, fever and a positive blood smear & $\operatorname{IRR}=1.09(95 \% \mathrm{Cl} 0.95-1.25), P=0.24$ & $\begin{array}{l}\text { No } \\
\text { impact }\end{array}$ \\
\hline & $<-2$ & Pf malaria, fever and a positive blood smear & $\operatorname{IRR}=1.12(95 \% \mathrm{Cl} 0.86-1.46), P=0.39$ & $\begin{array}{l}\text { No } \\
\text { impact }\end{array}$ \\
\hline Ayana 2015 [49] & $<-2$ & Malaria by RDT & "Non-significant association" reported & $\begin{array}{l}\text { No } \\
\text { impact }\end{array}$ \\
\hline $\begin{array}{l}\text { Custodio } 2009 \\
\text { [30] }\end{array}$ & $<-2$ & Pf malaria parasitemia prevalence & "Non-significant association" reported & $\begin{array}{l}\text { No } \\
\text { impact }\end{array}$ \\
\hline Deen 2002 [10] & $<-2$ & $\begin{array}{l}\text { Malaria episode (fever } \geq 37.5^{\circ} \mathrm{C} \text { or } \\
\text { parasitemia }>5000 / \mu \mathrm{L})\end{array}$ & $\mathrm{RR}=1.01(95 \% \mathrm{Cl}=0.82-1.26)$ & $\begin{array}{l}\text { No } \\
\text { impact }\end{array}$ \\
\hline $\begin{array}{l}\text { Deribew } 2010 \\
\text { [51] }\end{array}$ & $<-2$ & Pf malaria (any parasitemia) & $\mathrm{AOR}=0.9(95 \% \mathrm{Cl}=0.7-1.2), P=0.90$ & $\begin{array}{l}\text { No } \\
\text { impact }\end{array}$ \\
\hline \multirow{3}{*}{$\begin{array}{l}\text { Ehrhardt } 2006 \\
\text { [11] }\end{array}$} & $<-2$ & Fever & $\mathrm{AOR}=1.59(95 \% \mathrm{Cl}=1.13-2.23), P=0.008$ & Increased \\
\hline & $<-2$ & $\begin{array}{l}\text { Clinical malaria (fever } \geq 37.5^{\circ} \mathrm{C} \text { plus any } \\
\text { parasitemia) }\end{array}$ & $\mathrm{AOR}=1.67(95 \% \mathrm{Cl}=1.10-2.50), P=0.009$ & Increased \\
\hline & $<-2$ & Anaemia (Haemoglobin $<11$ g/dL) & $\mathrm{AOR}=1.68(95 \% \mathrm{Cl}=1.38-2.04), P<0.0001^{\varphi}$ & \\
\hline \multirow[t]{2}{*}{$\begin{array}{l}\text { El Samani } 1987 \\
\text { [52] }\end{array}$} & $\begin{array}{l}\text { Weight-for-age } 75-89 \% \\
\text { (mild) }\end{array}$ & History of malaria in past 2 months & $\mathrm{AOR}=1.20(95 \% \mathrm{Cl}=0.70-2.00)$ & $\begin{array}{l}\text { No } \\
\text { impact }\end{array}$ \\
\hline & $\begin{array}{l}\text { Weight-for-age }<75 \% \\
\text { (moderate) }\end{array}$ & History of malaria in past 2 months & $\mathrm{AOR}=2.10,(95 \% \mathrm{Cl}=1.10-4.00)$ & Increased \\
\hline \multirow[t]{2}{*}{ Fillol 2009 [27] } & $<-2$ & $\begin{array}{l}\text { Clinical malaria (fever } \geq 37.5^{\circ} \mathrm{C} \text { plus } \\
\text { parasitemia } \geq 3000 / \mu \mathrm{L} \text { ) }\end{array}$ & "Non-significant association" reported & $\begin{array}{l}\text { No } \\
\text { impact }\end{array}$ \\
\hline & $<-2$ & $\begin{array}{l}\text { High density parasitemia (geometric mean } \\
\geq 300 / \mu \mathrm{L} \text { ) }\end{array}$ & $\mathrm{AOR}=0.96(95 \% \mathrm{Cl}=0.35-2.66), P=0.94$ & $\begin{array}{l}\text { No } \\
\text { impact }\end{array}$ \\
\hline $\begin{array}{l}\text { Jeremiah } 2007 \\
\text { [53] }\end{array}$ & $<-2$ & Malaria by blood smear & $\mathrm{RR}=1.02(95 \% \mathrm{Cl}=0.34-2.37), P<0.02$ & Increased \\
\hline $\begin{array}{l}\text { Kateera } 2015 \\
{[54]}\end{array}$ & $<-2$ & Pf malaria (any parasitemia) & "Non-significant association" reported & $\begin{array}{l}\text { No } \\
\text { impact }\end{array}$ \\
\hline $\begin{array}{l}\text { Mitangala } 2013 \\
\text { [21] }\end{array}$ & $<-2$ & Pf malaria parasitemia ( $\geq 5000 / \mu \mathrm{L})$ & $\mathrm{AOR}=0.85(95 \% \mathrm{Cl}=0.53-1.35), P=0.49$ & $\begin{array}{l}\text { No } \\
\text { impact }\end{array}$ \\
\hline \multirow[t]{3}{*}{ Muller 2003 [18] } & $\leq-2$ & Pf malaria (fever plus parasitemia $\geq 1 / \mu \mathrm{L}$ ) & $\mathrm{RR}=1.0(95 \% \mathrm{Cl}=0.9-1.1), P=0.98$ & $\begin{array}{l}\text { No } \\
\text { impact }\end{array}$ \\
\hline & $\leq-2$ & $\begin{array}{l}\text { Pf malaria (fever plus parasitemia } \geq 5000 / \\
\mu \mathrm{L} \text { ) }\end{array}$ & $\mathrm{RR}=1.0(95 \% \mathrm{Cl}=0.9-1.2), P=0.68$ & $\begin{array}{l}\text { No } \\
\text { impact }\end{array}$ \\
\hline & $\leq-2$ & $\begin{array}{l}\text { Pf malaria (fever plus parasitemia } \geq 100,000 / \\
\mu \mathrm{L} \text { ) }\end{array}$ & $\mathrm{RR}=0.8(95 \% \mathrm{Cl}=0.5-1.4), P=0.49$ & $\begin{array}{l}\text { No } \\
\text { impact }\end{array}$ \\
\hline \multirow[t]{2}{*}{$\begin{array}{l}\text { Nyakeriga } 2004 \\
{[6]}\end{array}$} & $<-2$ & Pf malaria (fever plus any parasitemia) & $\mathrm{IRR}=1.33(95 \% \mathrm{Cl}=0.64-2.70), P=0.44$ & $\begin{array}{l}\text { No } \\
\text { impact }\end{array}$ \\
\hline & $<-2$ & $\begin{array}{l}\text { Pf malaria (fever plus any para }<1 \text { year, }> \\
2500 / \mu \mathrm{L}>1 \text { year) }\end{array}$ & $\mathrm{IRR}=0.28(95 \% \mathrm{Cl}=0.51-3.17), P=0.60$ & $\begin{array}{l}\text { No } \\
\text { impact }\end{array}$ \\
\hline \multirow[t]{2}{*}{ Snow 1991 [17] } & $<-2$ & Clinical malaria (fever plus any parasitemia) & "Non-significant association" reported & $\begin{array}{l}\text { No } \\
\text { impact }\end{array}$ \\
\hline & $<-2$ & Asymptomatic malaria parasitemia & "Non-significant association" reported & $\begin{array}{l}\text { No } \\
\text { impact }\end{array}$ \\
\hline \multirow{2}{*}{$\begin{array}{l}\text { Sumbele } 2015 \\
\text { [57] }\end{array}$} & $<-2$ & Clinical malaria parasitemia & Underweight vs. Non: $21.6 \%$ vs. $8.2 \%, P=0.007$ & Increased \\
\hline & $<-2$ & Asymptomatic malaria parasitemia & Underweight vs. Non: $21.6 \%$ vs. $27.5 \%, P=0.44$ & $\begin{array}{l}\text { No } \\
\text { impact }\end{array}$ \\
\hline
\end{tabular}


Table 4 Relationship between being underweight and risk of malarial infection ( $N=19)$ (Continued)

\begin{tabular}{|c|c|c|c|c|}
\hline $\begin{array}{l}\text { Author, year, } \\
\text { reference }\end{array}$ & WAZ cutoff & Malaria outcome & $\begin{array}{l}\text { Risk estimate comparing children below and } \\
\text { above WAZ cutoff }\end{array}$ & Risk \\
\hline \multirow[t]{2}{*}{$\begin{array}{l}\text { Tonglet } 1999 \\
\text { [32] }\end{array}$} & $<-2$ & $\begin{array}{l}\text { Clinical malaria without lab confirmation in } \\
<9 \mathrm{~m}\end{array}$ & $\mathrm{AOR}=1.31(95 \% \mathrm{Cl}=0.68-1.94)$ & $\begin{array}{l}\text { No } \\
\text { impact }\end{array}$ \\
\hline & $<-2$ & $\begin{array}{l}\text { Clinical malaria without lab confirmation in } \\
\geq 9 \mathrm{~m}\end{array}$ & $\mathrm{AOR}=0.68(95 \% \mathrm{Cl}=0.24-1.11)$ & $\begin{array}{l}\text { No } \\
\text { impact }\end{array}$ \\
\hline \multirow[t]{3}{*}{ Verret 2011 [22] } & $(\geq-1$ and $<0)$ & Pf malaria risk of recurrent parasitemia & $\mathrm{HR}=0.65(95 \% \mathrm{Cl}=0.37-1.15), P=0.137$ & $\begin{array}{l}\text { No } \\
\text { impact }\end{array}$ \\
\hline & $(\geq-2$ and $<-1)$ & Pf malaria risk of recurrent parasitemia & $\mathrm{HR}=0.86(95 \% \mathrm{Cl}=0.45-1.62), P=0.636$ & $\begin{array}{l}\text { No } \\
\text { impact }\end{array}$ \\
\hline & $<-2$ & Pf malaria risk of recurrent parasitemia & $\mathrm{HR}=1.01(95 \% \mathrm{Cl}=0.54-1.89), P=0.969$ & $\begin{array}{l}\text { No } \\
\text { impact }\end{array}$ \\
\hline \multirow[t]{4}{*}{$\begin{array}{l}\text { William } 1997 \\
\text { [31] }\end{array}$} & $<-2$ & $\begin{array}{l}\text { Clinical malaria (fever plus parasitemia } \geq \\
1000 / \mu \mathrm{L} \text { ) }\end{array}$ & $\operatorname{IRR}=1.1(95 \% \mathrm{Cl}=0.57-2.1)^{*}, P=0.8$ & $\begin{array}{l}\text { No } \\
\text { impact }\end{array}$ \\
\hline & $<-2$ & $\begin{array}{l}\text { Clinical malaria (fever plus parasitemia } \geq \\
1000 / \mu \mathrm{L} \text { ) }\end{array}$ & $\operatorname{IRR}=1.3(95 \% \mathrm{Cl}=0.9-1.9)^{* *}, P=0.2$ & $\begin{array}{l}\text { No } \\
\text { impact }\end{array}$ \\
\hline & $<-2$ & P. vivax malaria & $\operatorname{IRR}=2.6(95 \% \mathrm{Cl}=1.5-4.4)^{*}, P<0.0001$ & Increasec \\
\hline & $<-2$ & P. vivax malaria & $\operatorname{IRR}=1.3(95 \% \mathrm{Cl}=0.9-2.0)^{* *}, P=0.2$ & $\begin{array}{l}\text { No } \\
\text { impact }\end{array}$ \\
\hline
\end{tabular}

Pf Plasmodium falciparum, WAZ weight-for-age Z-scores, $C I$ confidence interval, OR odds ratio, $H R$ hazard ratio, $R R$ risk ratio, IRR incidence rate ratio, $A O R$ adjusted odds ratio

${ }^{\varphi}$ Limited to anaemia;*6 months preceding anthropometric assessment; ${ }^{* *} 6$ months following anthropometric assessment

HIV-infected and exposed infants) were at higher risk for recurrent parasitaemia (HR 3.18 (95\% CI 1.18-8.56); $P=$ 0.022). Another study carried out in the DRC in 445 children, comparing the efficacy of standard doses of artesunate-amodiaquine between children with and without severe acute malnutrition (SAM), observed no evidence of reduced efficacy in children with SAM, which had an adequate clinical and parasitological cure rate, ACPR, of 91.4\% [34]. A recent multi-centre (Mali and Niger), open-label trial compared the efficacy and pharmacokinetics of artemether-lumefantrine in 399 children with or without SAM. The results of this study showed adequate therapeutic efficacy in both SAM and non-SAM groups (day 42 ACPR $100 \%$ vs. $98.3 \%$ respectively) with no early treatment failures and no difference in parasite clearance reported. However, a higher risk of reinfection in children older than 21 months suffering from SAM was evident (AHR 2.10 (1.04-4.22); $P=0.038$ ) [35]. Similarly in a large pooled analysis of individual pharmacokinetic-pharmacodynamic (PK-PD) data from 2787 patients treated with artemether-lumefantrine for uncomplicated $P f$ malaria, the WorldWide Antimalarial Resistance Network (WWARN) demonstrated that among children 1-4 years of age in high-transmission areas, the risk of reinfection increased with a decrease in WAZ with a HR of 1.63 (95\% CI 1.09 to 2.44) for a child with WAZ of -3 compared to an adequately nourished child $(\mathrm{WAZ}=0)$ [36].

Information on the pharmacokinetic properties of ACTs in malnourished children is critically lacking in the published literature. Our search retrieved a study published in 2016 which assessed the efficacy of AL in relation to drug exposure in children with SAM vs. non-SAM in Mali and Niger [35]. This study measured lumefantrine concentration and showed that despite the administration of $92 \mathrm{~g}$ fat with dosing of SAM children (compared to $15 \mathrm{~mL}$ milk in non-SAM children), day 7 lumefantrine concentrations were lower in children with SAM compared to non-SAM (median 251 vs. $365 \mathrm{ng} / \mathrm{mL}, P=0.049$ ). In the WWARN pooled analysis of individual PK-PD data from patients treated with artemether-lumefantrine for uncomplicated $P f$ malaria, underweight-for-age young children $(<3$ years) had $23 \%$ (95\% CI -1 to $41 \%$ ) lower day 7 lumefantrine concentrations than adequately nourished children of same age [36].

\section{Discussion}

The evidence on the effect of malnutrition on malaria risk remains controversial and in many instances contradictory. The current review highlights some key limitations in the way the interaction between malaria and malnutrition has been assessed and reported. First, differences in methodology, study populations, the variability in measures used to define malnutrition (e.g. different growth references, different cut off thresholds), and the heterogeneous malaria transmission intensities with different levels of host immunity within the different studies make the comparison challenging. Second, there is a paucity of information on the effect of malnutrition on therapeutic responses to ACTs and their pharmacokinetic properties in malnourished children in published literature. Generally, vulnerable populations 
with common co-morbidities such as malnutrition, obesity, HIV or tuberculosis co-infection are excluded from or under-represented in antimalarial drug efficacy trials [37]. Although weight is documented, height is rarely recorded in ACT efficacy trials $(<20 \%$ of 250 trials currently included in the WWARN repository, personal communication Kasia Stepniewska), restricting the possibilities for secondary analyses. Another useful metric, mid-upper arm circumference (MUAC) is also rarely documented in malaria clinical trials despite being relatively easy to measure and low MUAC shown to be associated with increased malaria risk [38] and decreased lumefantrine bioavailability [39]. Several confounding factors and effect modifiers have been suggested such as age, co-morbidities (e.g. HIV, tuberculosis co-infection and drug interactions), immunity, socio-economic status, or refeeding practices. However, these confounding factors are poorly documented and controlled for in most of the reported studies in this review.

This review has several limitations. First, one third of the studies included in this review recruited individuals of all ages, and disaggregating observations by the age of individuals (below and above 5 years) was not possible. Methodologically, the temporal relationship between malnutrition and risk of malaria (and progression from infection to symptomatic malaria) could not be assessed because of cross-sectional study design ( $50 \%$ included studies). It is also limited by the extent to which important confounders (such as differential micronutrient deficiencies, ecological and genetic factors) are measured and reported in the included articles. Finally, the heterogeneity of the selected studies (presented as Additional file 5) including variations in measurement of nutritional status, definition of malaria, and statistical approaches adopted in deriving the risk estimates restricted plausible aggregated data meta-analysis in this review.

Interestingly, while no consistent association between risk of malaria and acute malnutrition was found, chronic malnutrition was relatively consistently associated with severity of malaria such as high-density parasitemia and anaemia $[10,26,27]$. The mechanism behind the higher risk of recurrent parasitemia could be explained partially by the impact of chronic malnutrition on the immune system and/or lower antimalarial bioavailability. Likewise, the apparent protection of wasted children from clinical malaria might be caused by their being administered a higher $\mathrm{mg} / \mathrm{kg}$ antimalarial dose and/or a modulation of their immune response and thus an absence of symptoms, e.g. fever usually associated with malaria as opposed to an absence of malaria infection. Friedman et al. showed high-density parasitemia as a predictor for chronic malnutrition [26]. Nevertheless, the role of malaria in the aetiology of malnutrition remains unclear. The effect of malaria on nutritional status appeared to be greatest during the first 2 years of life and age acted as an effect modifier in the association between malaria episodes and malnutrition [6].

ACTs are now recommended for the treatment of uncomplicated falciparum malaria in almost all malaria-endemic countries and the number of children exposed to these antimalarial agents is increasing. A priority area is to identify gaps in our current knowledge in regard to the pharmacokinetic properties of artemisinins and partner drugs in malnourished paediatric populations to optimise dosing in order to ensure efficacy, safety and avoid the selection of parasite resistance [40]. Exposing pathogens to sub-therapeutic levels of active ingredients is a major driver of resistance. Protein-energy malnutrition, defined as insufficient calorie and protein intake, may have potential physiological effects on the absorption, distribution and metabolism of ACTs and subsequently affect the efficacy and safety of ACTs. Severe acute malnutrition can cause pathophysiological changes, including increasing total body water, leading to greater volume of distribution of drugs, which in turn may cause sub-optimal drug exposure when ACTs are given at standard doses [35, 41]. This could be further compounded by malnutrition in paediatric patients leading to dosing inaccuracies of ACTs when dose is calculated by age (over-dosing for actual body weight) or weight (under-dosing for age). Malnutrition can also be associated with intestinal malabsorption and villous atrophy of the jejunal mucosa which may cause impaired drug absorption [42]. The reduced absorption of lipids and fats has the potential to specifically affect the lipid-soluble ACTs [43]. The hepatic metabolism of the ACTs may be compromised in malnourished children. For instance, in case of quinine, hepatic metabolism is decreased in protein deficiency and increased in global malnutrition [44]. Thus, hepatic metabolised drugs should be carefully monitored in children with malnutrition. A recent individual patient data (IPD) meta-analysis conducted by the WWARN to investigate the effect of dosing strategy on efficacy of artemether-lumefantrine showed that the risk of treatment failure was greatest in malnourished children aged 1-3 years in Africa (PCR-adjusted efficacy 94.3\%, 95\% CI 92.3-96.3) [45]. Another large WWARN IPD meta-analysis of individual PK-PD data from patients treated with artemether-lumefantrine (AL) for uncomplicated malaria, showed day 7 concentrations adjusted for $\mathrm{mg} / \mathrm{kg}$ dose were lowest in very young children $(<3$ years), among whom underweight-for-age children had 23\% (95\% $\mathrm{CI}-1$ to $41 \%$ ) lower concentrations than adequately nourished children of the same age and 53\% (95\% CI 37 to 65\%) lower concentrations than adults [36]. This raises the question of whether an adapted dosing regimen is needed in malnourished young children. The PK-PD evaluation of artemisinins and longer-acting partner antimalarials for the treatment of malaria in paediatric populations and the 
effect of malnutrition on the pharmacological activity of ACTs is a priority area to identify and address key knowledge gaps.

\section{Conclusions}

A summary of the remaining knowledge gaps is presented to serve as the basis for prioritising future research strategies and highlights the need for standardising measures and reporting of nutritional status. Further analyses using individual patient data could provide an important opportunity to better understand the variability observed in publications by standardising both malaria nutritional metrics. In an era of emergence and spread of antimalarial drug resistance, it is imperative to improve our understanding of the pharmacodynamics and pharmacokinetics of ACTs in malnourished children to optimise antimalarial treatment of this very large vulnerable population. Pooled analysis, gap analysis and carefully designed prospective, randomised controlled clinical trials can provide strong evidence on the outstanding questions raised in this review related to malaria-malnutrition interactions.

\section{Additional files}

Additional file 1: PubMed, Global Health, Cochrane database search terms (DOCX $15 \mathrm{~kb}$ )

Additional file 2: PRISMA checklist (DOC $67 \mathrm{~kb}$ )

Additional file 3: The methodological quality of the included studies (DOCX $33 \mathrm{~kb}$ )

Additional file 4: Characteristics of the selected studies ( $N=33)(X L S X 12 \mathrm{~kb})$

Additional file 5: Graphical presentation of reported risk estimates by nutritional status (DOCX $135 \mathrm{~kb}$ )

\section{Abbreviations}

ACT: Artemisinin combination therapy; AOR: Adjusted odds ratio; Cl: Confidence interval; DRC: Democratic Republic of Congo; GRADE: Grading of Recommendations Assessment, Development, and Evaluation; HAZ: Height-for-age Z-score; HR: Hazard ratio; IPD: Individual patient data; IRR: Incidence risk ratio; MUAC: Mid-Upper Arm Circumference; NOS: Newcastle-Ottawa Scale; OR: Odds ratio; Pf: Plasmodium falciparum; PK-PD: Pharmacokinetic-pharmacodynamic; RR: Risk ratio; SAM: Severe acute malnutrition; SD: Standard deviation; WAZ: Weight-for-age Z-score; WHO: World Health Organization; WHZ: Weight-for-height Z-score; WWARN: WorldWide Antimalarial Resistance Network

\section{Acknowledgements}

The authors gratefully acknowledge the assistance of Eli Harriss with database searches and Per Olav Vandvik for reviewing the methods of the manuscript. The authors are also very grateful to Prabin Dahal, Makoto Saito, Andrea Stewart and Alex Gardiner for their valuable comments on the manuscript.

\section{Funding}

WorldWide Antimalarial Resistance Network (WWARN) is funded by the Bill and Melinda Gates Foundation. EAO is supported by the Wellcome as an Intermediary Fellow (\# 201866) and also acknowledge the support of the Wellcome to the Kenya Major Overseas Programme (\# 077092 and 203077).

\section{Availability of data and materials}

All the data used in this review has been presented in the main text either as tables or as supplementary files. Additional requests for the data can be made through the WorldWide Antimalarial Resistance Network

(www.wwarn.org). Please email: clinical@wwarn.org

\section{Authors' contributions}

PJG conceived the concept of the review and supervised and coordinated the project. DD and RM reviewed literatures and extracted data. KS and RM reviewed the methodology and data analysis. DD drafted the manuscript. $\mathrm{KIB}, \mathrm{RFG}, \mathrm{EO}, \mathrm{SK}, \mathrm{KS}$ and PJG reviewed and edited drafts of the manuscript. DJT and JT provided intellectual input on the review. All authors read and approved the final version of the manuscript.

Ethics approval and consent to participate

Not applicable.

\section{Consent for publication}

Not applicable.

\section{Competing interests}

The authors declare that they have no competing interests.

\section{Publisher's Note}

Springer Nature remains neutral with regard to jurisdictional claims in published maps and institutional affiliations.

\section{Author details}

${ }^{1}$ WorldWide Antimalarial Resistance Network (WWARN), Oxford, UK. ${ }^{2}$ Centre for Tropical Medicine and Global Health, Nuffield Department of Clinical Medicine, University of Oxford, Oxford, UK. ${ }^{3}$ Mahidol Oxford Tropical Medicine Research Unit, Faculty of Tropical Medicine, Mahidol University, Bangkok, Thailand. ${ }^{4}$ Epicentre, Paris, France. ${ }^{5}$ Médecins Sans Frontières, Amsterdam, Netherlands. ${ }^{6}$ Liverpool School of Tropical Medicine, Liverpool, UK. ${ }^{7}$ Kemri Wellcome Trust Research Programme, Kilifi, Kenya. ${ }^{8}$ Division of Clinical Pharmacology, Department of Medicine, University of Cape Town, Cape Town, South Africa. ${ }^{9}$ WorldWide Antimalarial Resistance Network (WWARN) Pharmacology, University of Cape Town, Cape Town, South Africa.

${ }^{10}$ Malawi-Liverpool Wellcome Trust Clinical Research Programme, Blantyre,

Malawi. ${ }^{11}$ College of Medicine, University of Malawi, Blantyre, Malawi.

Received: 24 April 2018 Accepted: 20 September 2018

Published online: 29 October 2018

\section{References}

1. World Malaria Report 2016. Geneva: World Health Organization; 2016. http:// www.who.int/malaria/publications/world-malaria-report-2016/report/en/.

2. Black RE, Victora CG, Walker SP, Bhutta ZA, Christian $P$, de Onis M, Ezzati M, Grantham-McGregor S, Katz J, Martorell R, et al. Maternal and child undernutrition and overweight in low-income and middle-income countries. Lancet. 2013;382(9890):427-51.

3. WHOMGRS. WHO child growth standards based on length/height, weight and age. Acta Paediatr Suppl. 2006:450:76-85.

4. Bradley-Moore AM, Greenwood BM, Bradley AK, Kirkwood BR, Gilles HM. Malaria chemoprophylaxis with chloroquine in young Nigerian children. III Its effect on nutrition. Ann Trop Med Parasitol. 1985;79(6):575-84.

5. Colbourne MJ. The effect of malaria suppression in a group of Accra school children. Trans R Soc Trop Med Hyg. 1955;49(4):556-69.

6. Nyakeriga AM, Troye-Blomberg M, Chemtai AK, Marsh K, Williams TN. Malaria and nutritional status in children living on the coast of Kenya. Am J Clin Nutr. 2004;80(6):1604-10.

7. Snow RW, Molyneux CS, Njeru EK, Omumbo J, Nevill CG, Muniu E, Marsh K. The effects of malaria control on nutritional status in infancy. Acta Trop. 1997; 65(1):1-10.

8. ter Kuile FO, Terlouw DJ, Kariuki SK, Phillips-Howard PA, Mirel LB, Hawley WA, Friedman JF, Shi YP, Kolczak MS, Lal AA, et al. Impact of permethrintreated bed nets on malaria, anemia, and growth in infants in an area of intense perennial malaria transmission in western Kenya. Am J Trop Med Hyg. 2003;68(4 Suppl):68-77.

9. ter Kuile FO, Terlouw DJ, Phillips-Howard PA, Hawley WA, Friedman JF, Kolczak MS, Kariuki SK, Shi YP, Kwena AM, Vulule JM, et al. Impact of 
permethrin-treated bed nets on malaria and all-cause morbidity in young children in an area of intense perennial malaria transmission in western Kenya: cross-sectional survey. Am J Trop Med Hyg. 2003;68(4 Suppl):100-7.

10. Deen $J$, Walraven GEL, von Seidlein L. Increased risk for malaria in chronically malnourished children under 5 years of age in rural Gambia. J Trop Pediatr. 2002:48(2):78-83.

11. Ehrhardt S, Burchard GD, Mantel C, Cramer JP, Kaiser S, Kubo M, Otchwemah RN, Bienzle U, Mockenhaupt FP. Malaria, anemia, and malnutrition in african children--defining intervention priorities. J Infect Dis. 2006;194(1):108-14.

12. Man WD, Weber M, Palmer A, Schneider G, Wadda R, Jaffar S, Mulholland EK, Greenwood BM. Nutritional status of children admitted to hospital with different diseases and its relationship to outcome in the Gambia, West Africa. Tropical Med Int Health. 1998;3(8):678-86.

13. Verhoef H, West CE, Veenemans J, Beguin Y, Kok FJ. Stunting may determine the severity of malaria-associated anemia in African children. Pediatrics. 2002;110(4):e48.

14. Genton B, Al-Yaman F, Ginny M, Taraika J, Alpers MP. Relation of anthropometry to malaria morbidity and immunity in Papua new Guinean children. Am J Clin Nutr. 1998;68(3):734-41.

15. Hendrickse RG, Hasan AH, Olumide LO, Akinkunmi A. Malaria in early childhood. An investigation of five hundred seriously ill children in whom a "clinical" diagnosis of malaria was made on admission to the children's emergency room at University College Hospital, Ibadan. Ann Trop Med Parasitol. 1971;65(1):1-20.

16. Murray MJ, Murray AB, Murray NJ, Murray MB. Diet and cerebral malaria: the effect of famine and refeeding. Am J Clin Nutr. 1978;31(1):57-61.

17. Snow RW, Byass P, Shenton FC, Greenwood BM. The relationship between anthropometric measurements and measurements of iron status and susceptibility to malaria in Gambian children. Trans R Soc Trop Med Hyg. 1991;85(5):584-9.

18. Muller $\mathrm{O}$, Garenne M, Kouyate B, Becher $\mathrm{H}$. The association between protein-energy malnutrition, malaria morbidity and all-cause mortality in west African children. Tropical Med Int Health. 2003;8(6):507-11.

19. Tshikuka JG, Gray-Donald K, Scott M, Olela KN. Relationship of childhood protein-energy malnutrition and parasite infections in an urban African setting. Tropical Med Int Health. 1997;2(4):374-82.

20. Hess Fl, Nukuro E, Judson L, Rodgers J, Nothdurft HD, Rieckmann KH. Antimalarial drug resistance, malnutrition and socio-economic status. Tropical Med Int Health. 1997;2(8):721-8.

21. Mitangala PN, D'Alessandro U, Donnen P, Hennart P, Porignon D, Bisimwa Balaluka G, Zozo Nyarukweba D, Cobohwa Mbiribindi N, Dramaix Wilmet M. Malaria infection and nutritional status: results from a cohort survey of children from 6-59 months old in the Kivu province, Democratic Republic of the Congo. Revue d'epidemiologie et de sante publique. 2013;61(2):111-20.

22. Verret WJ, Arinaitwe E, Wanzira H, Bigira V, Kakuru A, Kamya M, Tappero JW, Sandison T, Dorsey G. Effect of nutritional status on response to treatment with artemisinin-based combination therapy in young Ugandan children with malaria. Antimicrob Agents Chemother. 2011;55(6):2629-35.

23. Moher D, Liberati A, Tetzlaff J, Altman DG, Group P. Preferred reporting items for systematic reviews and meta-analyses: the PRISMA statement. BMJ (Clinical research ed). 2009;339:b2535.

24. Well GASB, O'Connell D, Peterson J. The Newcastle-Ottawa scale (NOS) for assessing the quality of non-randomised studies in meta-analyses. Ottawa: Ottawa Hospital Research Institute; 2011.

25. Guyatt GHOA, Vist G, Kunz R, Brozek J. GRADE guidelines: 4. Rating the quality of evidence--study limitations (risk of bias). J Clin Epidemiol. 2011;64(4):407-15.

26. Friedman JF, Kwena AM, Mirel LB, Kariuki SK, Terlouw DJ, Phillips-Howard PA, Hawley WA, Nahlen BL, Shi YP, ter Kuile FO. Malaria and nutritional status among pre-school children: results from cross-sectional surveys in western Kenya. Am J Trop Med Hyg. 2005;73(4):698-704.

27. Fillol F, Cournil A, Boulanger D, Cisse B, Sokhna C, Targett G, Trape JF, Simondon F, Greenwood B, Simondon KB. Influence of wasting and stunting at the onset of the rainy season on subsequent malaria morbidity among rural preschool children in Senegal. Am J Trop Med Hyg. 2009;80(2):202-8.

28. Shikur B, Deressa W, Lindtjørn B. Association between malaria and malnutrition among children aged under-five years in Adami Tulu District, south-Central Ethiopia: a case-control study. BMC Public Health. 2016;16(1):174.

29. Takakura M, Uza M, Sasaki Y, Nagahama N, Phommpida S, Bounyadeth S, Kobayashi J, Toma T, Miyagi I. The relationship between anthropometric indicators of nutritional status and malaria infection among youths in Khammouane Province, Lao PDR. Southeast Asian J Trop Med Public Health. 2001;32(2):262-7.
30. Custodio E, Descalzo MA, Villamor E, Molina L, Sanchez I, Lwanga M, Bernis C, Benito A, Roche J. Nutritional and socio-economic factors associated with Plasmodium falciparum infection in children from Equatorial Guinea: results from a nationally representative survey. Malar J. 2009;8:225.

31. Williams TN, Maitland K, Phelps L, Bennett S, Peto TE, Viji J, Timothy R, Clegg $J B$, Weatherall DJ, Bowden DK. Plasmodium vivax: a cause of malnutrition in young children. QJM. 1997;90(12):751-7.

32. Tonglet R, Mahangaiko Lembo E, Zihindula PM, Wodon A, Dramaix M, Hennart P. How useful are anthropometric, clinical and dietary measurements of nutritional status as predictors of morbidity of young children in Central Africa? Trop Med Int Health. 1999;4(2):120-30.

33. Obua C, MNJWO-OLLGUH, Petzold MG. Impact of nutritional status on fixed-dose chloroquine and sulfadoxine/pyrimethamine combination treatment of malaria in Ugandan children. Int J Trop Med. 2008;3(3):53-9.

34. Mitanlgala NP, D'Alessandro U, Hennart P, Donnen P, Porignon D. Efficacy of Artesunate plus Amodiaquine for treatment of uncomplicated clinical falciparum malaria in severely malnourished children aged 6-59 months, Democratic Republic of Congo. J Clin Exp Pathol. 2012;S3:005.

35. Denoeud-Ndam L, Dicko A, Baudin E, Guindo O, Grandesso F, Diawara H, Sissoko S, Sanogo K, Traoré S, Keita S, et al. Efficacy of artemetherlumefantrine in relation to drug exposure in children with and without severe acute malnutrition: an open comparative intervention study in Mali and Niger. BMC Med. 2016;14(1):167.

36. WorldWide Antimalarial Resistance Network Lumefantrine PKPDSG. Artemether-lumefantrine treatment of uncomplicated Plasmodium falciparum malaria: a systematic review and meta-analysis of day 7 lumefantrine concentrations and therapeutic response using individual patient data. BMC Med. 2015;13:227.

37. Barnes Kl, Lindegardh N, Ogundahunsi O, Olliaro P, Plowe CV, Randrianarivelojosia M, Gbotosho GO, Watkins WM, Sibley CH, White NJ. World antimalarial resistance network (WARN) IV: clinical pharmacology. Malar J. 2007;6:122.

38. Gahutu J-B, Steininger C, Shyirambere C, Zeile I, Cwinya-Ay N, Danquah I, Larsen CH, Eggelte TA, Uwimana A, Karema C, et al. Prevalence and risk factors of malaria among children in southern highland Rwanda. Malar J. 2011;10(1):134.

39. Chotsiri P, Denoeud-Ndam L, Charunwatthana P, Dicko A: Population pharmacokinetic and time-to-event modelling of the antimalarial drug lumefantrine in young children with severe acute malnutrition. In: Available from: https://www.page-meeting.org/pdf_assets/6574-20170608_PAGE_ poster_LF_SAM_children_print.pdf.

40. Mercer AE, Sarr Sallah M. The pharmacokinetic evaluation of artemisinin drugs for the treatment of malaria in paediatric populations. Expert Opin Drug Metab Toxicol. 2011;7(4):427-39.

41. Oshikoya KA, Sammons HM, Choonara I. A systematic review of pharmacokinetics studies in children with protein-energy malnutrition. Eur J Clin Pharmacol. 2010;66(10):1025-35.

42. Oshikoya KA, Senbanjo IO. Pathophysiological changes that affect drug disposition in protein-energy malnourished children. Nutr Metab. 2009:6:50.

43. Murphy JL, Badaloo AV, Chambers B, Forrester TE, Wootton SA, Jackson AA. Maldigestion and malabsorption of dietary lipid during severe childhood malnutrition. Arch Dis Child. 2002;87(6):522-5.

44. Treluyer JM, Roux A, Mugnier C, Flouvat B, Lagardere B. Metabolism of quinine in children with global malnutrition. Pediatr Res. 1996;40(4):558-63.

45. Worldwide Antimalarial Resistance Network ALDISG. The effect of dose on the antimalarial efficacy of artemether-lumefantrine: a systematic review and pooled analysis of individual patient data. Lancet Infect Dis. 2015;15(6): 692-702.

46. Akiyama T, Pongvongsa $T$, Phrommala $S$, Taniguchi $T$, Inamine $Y$, Takeuchi $R$, Watanabe T, Nishimoto F, Moji K, Kano S, et al. Asymptomatic malaria, growth status, and anaemia among children in Lao People's Democratic Republic: a cross-sectional study. Malar J. 2016;15(1):499.

47. Alexandre MAA, Benzecry SG, Siqueira AM, Vitor-Silva S, Melo GC, Monteiro WM, Leite HP, Lacerda MVG, Alecrim MGC. The association between nutritional status and malaria in children from a rural community in the Amazonian region: a longitudinal study. PLoS Negl Trop Dis. 2015;9(4): e0003743.

48. Arinaitwe E, Gasasira A, Verret W, Homsy J, Wanzira H, Kakuru A, Sandison TG, Young S, Tappero JW, Kamya MR, et al. The association between malnutrition and the incidence of malaria among young HIV-infected and -uninfected Ugandan children: a prospective study. Malar J. 2012;11(1):90. 
49. Ayana TG, Solomon T, Atsbeha H, Shumbulo EL, Amente WD, Lindtjorn B. Malnutrition and malaria infection in children 6-59 months: a cohort study in Adami Tulu District, Ethiopia. Trop Med Int Health. 2015;20(Suppl. 1):294.

50. Crookston BT, Alder SC, Boakye I, Merrill RM, Amuasi JH, Porucznik CA, Stanford JB, Dickerson TT, Dearden KA, Hale DC, et al. Exploring the relationship between chronic undernutrition and asymptomatic malaria in Ghanaian children. Malar J. 2010;9(1):39.

51. Deribew A, Alemseged F, Tessema F, Sena L, Birhanu Z, Zeynudin A, Sudhakar M, Abdo N, Deribe K, Biadgilign S. Malaria and under-nutrition: a community based study among under-five children at risk of malaria, southWest Ethiopia. PLoS One. 2010;5(5):e10775.

52. el Samani FZ, Willett WC, Ware JH. Nutritional and socio-demographic risk indicators of malaria in children under five: a cross-sectional study in a Sudanese rural community. J Trop Med Hyg. 1987;90(2):69-78.

53. Jeremiah ZA, Uko EK. Childhood asymptomatic malaria and nutritional status among Port Harcourt children. East Afr J Public Health. 2007;4(2):55-8.

54. Kateera F, Ingabire CM, Hakizimana E, Kalinda P, Mens PF, Grobusch MP, Mutesa L, van Vugt M. Malaria, anaemia and under-nutrition: three frequently co-existing conditions among preschool children in rural Rwanda. Malar J. 2015;14(1):440.

55. Maketa V, Mavoko HM, da Luz RI, Zanga J, Lubiba J, Kalonji A, Lutumba P, Van Geertruyden J-P. The relationship between Plasmodium infection, anaemia and nutritional status in asymptomatic children aged under five years living in stable transmission zones in Kinshasa, Democratic Republic of Congo. Malar J. 2015;14(1):83.

56. Mamiro PS, Kolsteren P, Roberfroid D, Tatala S, Opsomer AS, Van Camp JH. Feeding practices and factors contributing to wasting, stunting, and irondeficiency anaemia among 3-23-month old children in Kilosa district, rural Tanzania. J Health Popul Nutr. 2005;23(3):222-30.

57. Sumbele IUN, Bopda OSM, Kimbi HK, Ning TR, Nkuo-Akenji T. Nutritional status of children in a malaria meso endemic area: cross sectional study on prevalence, intensity, predictors, influence on malaria parasitaemia and anaemia severity. BMC Public Health. 2015;15(1):1099.

58. Uscategui Penuela RM, Perez Tamayo EM, Corrales Agudelo LV, Correa Botero A, Estrada Restrepo A, Carmona Fonseca J. Relationship between malaria, malnutrition, food insecurity and low socioeconomical conditions in children of Turbo, Colombia. Perspectivas en Nutricion Humana. 2009;11:153-64.

Ready to submit your research? Choose BMC and benefit from:

- fast, convenient online submission

- thorough peer review by experienced researchers in your field

- rapid publication on acceptance

- support for research data, including large and complex data types

- gold Open Access which fosters wider collaboration and increased citations

- maximum visibility for your research: over $100 \mathrm{M}$ website views per year

At BMC, research is always in progress.

Learn more biomedcentral.com/submissions 